\title{
Das AO Faculty Education Program (FEP)
}

Klaus Dresing, Philip Wilbrandt

Die AO schult ihre Faculty, um eine höchstmögliche Qualität der Kurse sicherzustellen. Für die Schulung der AO Faculty gibt es ein Faculty Education Program (FEP), für Kursleiter ein Chairmen Training Program (CTP), ein Leaders Education Program (LEP). In Deutschland werden 2 FEP-Kurse pro Jahr angeboten. Zusätzlich gibt es individuelles Coaching für Faculty auf ausgewählten Kursen. Die Kurse des CTPs und LEPs finden bisher auf internationaler Ebene statt.

Das Faculty Education Program besteht aus 3 Teilen:

- Onlinekurs mit 5 Modulen zur Vorbereitung

- 11⁄2-tägiger Kurs, während dem jede(r) Teilnehmer(in) einen Vortrag halten, eine Diskussionsrunde leiten und eine praktische Übung betreuen muss. Der Vortrag wird aufgezeichnet. Im Vordergrund steht konstruktives Feedback mit konkreten Verbesserungsvorschlägen, das sowohl individuell als auch in der Gruppe erarbeitet wird.

- Online Nachbereitung
In 2017 finden die FEPs am 16./17. Juni und 10./11. November in Göttingen im Sartorius College statt.

Teilnahmevoraussetzungen sind:

- Teilnahme am AOTrauma-Prinzipienkurs und -Fortgeschrittenenkurs

- AOTrauma-Mitglied

- Referenten- oder Instruktoreinsatz bei AO-Kursen oder der Einsatz ist innerhalb der kommenden 6-12 Monate geplant.

Bewerbungen sind zu richten an Prof. Dr. Florian Gebhard (Ulm), Vorsitzender der AOT EDUC Deutschland, E-Mail: Florian.Gebhard@uniklinik-ulm.de

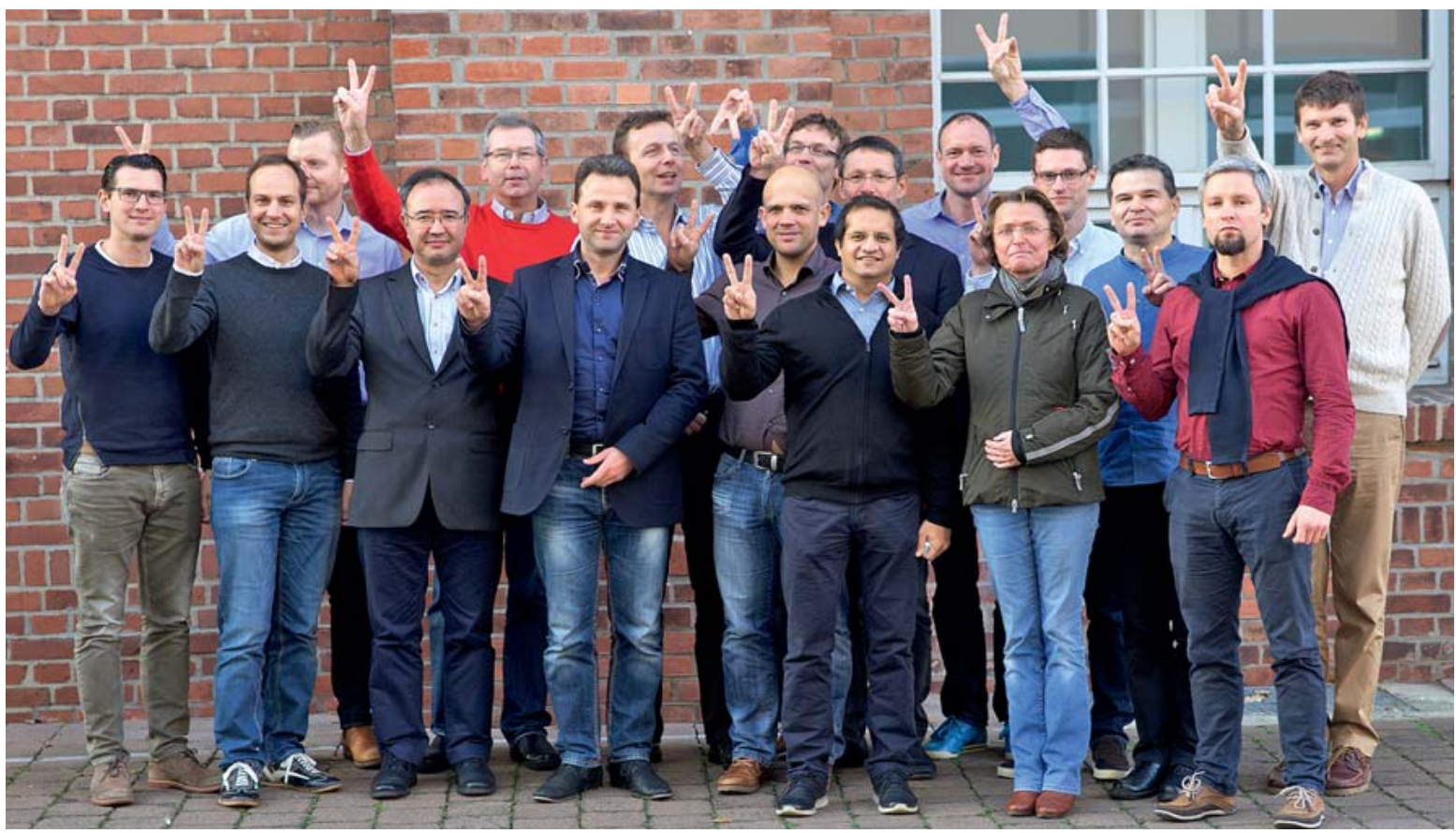

- Abb. 1 Teilnehmer des FEPs im November 2016 in Göttingen. Bild: Klaus Dresing. 


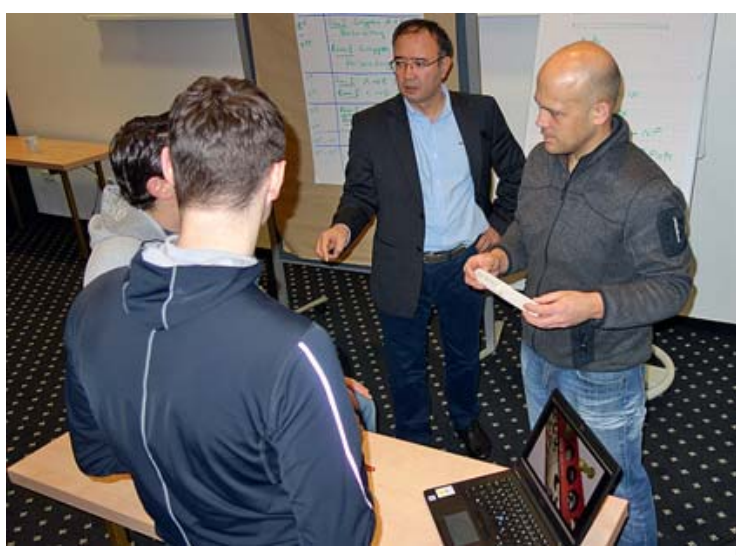

- Abb. 2 Arbeit in Kleingruppen. Bild: Klaus Dresing.

\section{Beurteilungen von Teilnehmern/ Teilnehmerinnen}

\section{Dr. Sven Weisser (Tübingen)}

„Das FEP-Konzept ist einmalig - keine langweiligen Vorlesungen, in denen man erzählt bekommt, wie man seinen Lehrstil verbessern könnte, sondern praktische Übungen und anschließende anregende Diskussionen. Es wurde individuell auf jeden Teilnehmer eingegangen und ihm Tipps, Lob und ,Kritik' gegeben - wobei die ,Kritik“ nicht wie gewohnt ausgeführt wurde, sondern die positiven Aspekte gefördert wurden und Anregungen gemacht wurden, was man das nächste Mal anders machen würde. Für mich eine neue Erfahrung in der deutschen Feedbackmentalität. Diesen Kurs kann ich jedem empfehlen, da man sich persönlich weiterentwickelt. Kurz zusammengefasst: AO Spirit live.“

\section{Dr. Steffen Schwarz (Freiburg)}

„Es war ein sehr guter Kurs mit einer Vielzahl an guten Tipps und praktischen Anleitungen. Bei sensiblen Themen wie z.B. Feedbackgabe in einer Fortbildung erfuhr man hilfreiche und konkrete Unterstüt-

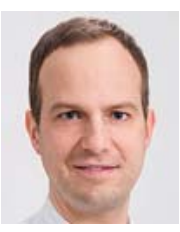
zung, welche in den praktischen Übungen direkt umgesetzt werden konnte. Ein konstruktives Feedback zeigte sich hier wesentlich effektiver und für alle Beteiligten angenehmer als ein bisher häufig praktiziertes ,Fehleraufzeigen“. Das gemeinsame Erleben der verschiedenen, zum Teil auch schwierigen Situationen bei Vorträgen, Diskussionsrunden und praktischen Übungen im Rahmen einer Fortbildung vermittelte eine zunehmende Sicherheit in der Tätigkeit als Faculty.“

\section{Dr. Dr. Philipp Poxleitner (Freiburg)}

„Als klinisch tätige Ärzte sind wir nicht in erster Linie Lehrer und auch nicht entsprechend geschult. Die Teilnahme am

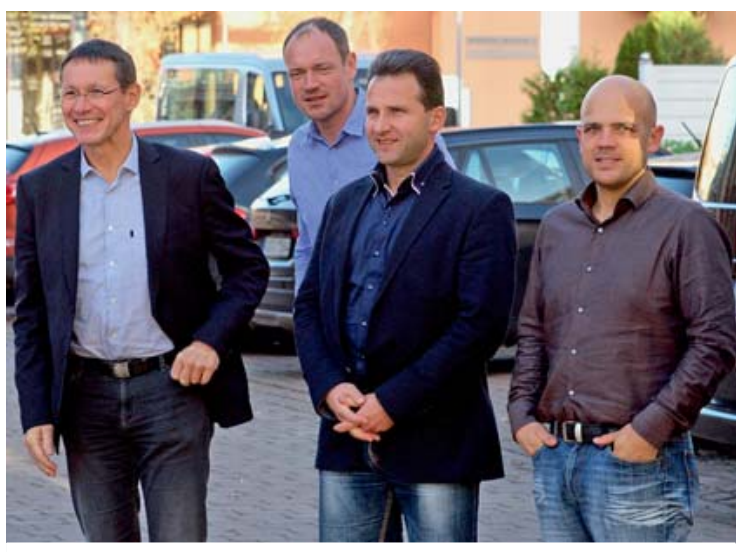

Abb. 3 Laier, Maier, König und Weisser. Bild: Klaus Dresing.

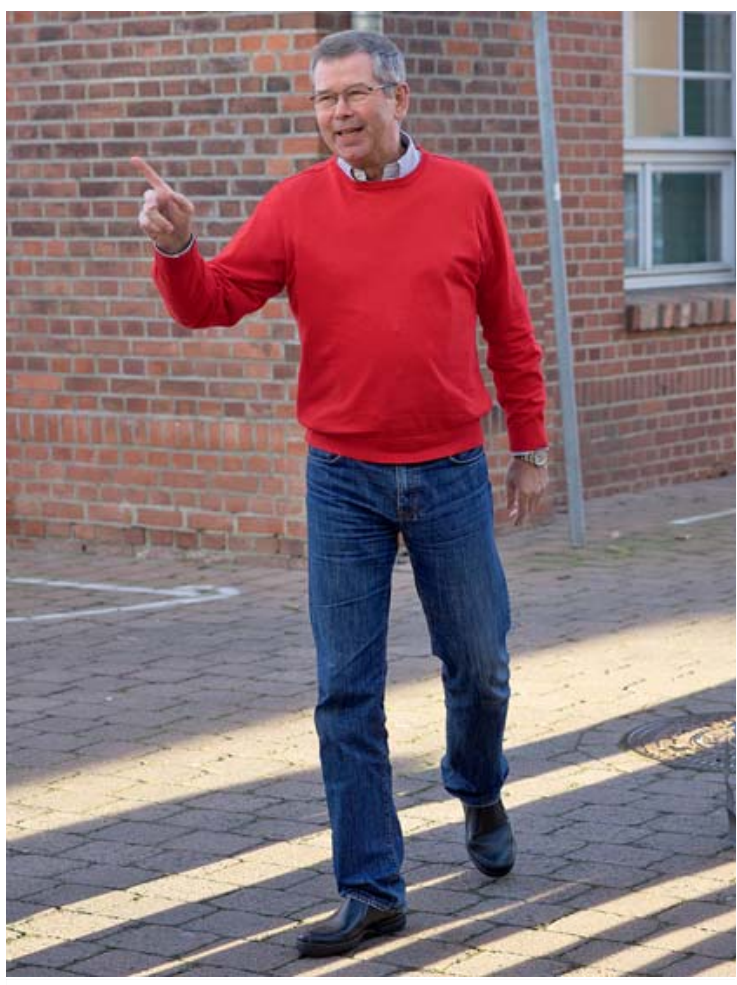

Abb. 4 Kursleiter Klaus Dresing. Bild: Klaus Dresing.

Faculty Educational Program gab mir die Möglichkeit, die verschiedenen Lehrkonzepte kennenzulernen und praktisch anzuwenden. Dabei wurde Wert auf die Qualität und Präsentation von Vorträgen, das Führen von Kleingruppendiskussionen und praktischen Übungen gelegt. Insbesondere das strukturierte Feedbackgeben hat mir für meine tägliche Arbeit viel gebracht.“ 


\section{Dr. Benjamin König (Freudenstadt)}

„Der FEP-Kurs hat meinen Wissensstand zum Lehren Erwachsener sowie meine Befähigung Lerninhalte zu vermitteln, deutlich erweitert. Er hilft sehr, die in der Medizinausbildung verbliebenen Defizite zur Arbeit mit Erwachsenen auszugleichen. Neben der Verbesserung meiner Präsentationstechnik und der Übung Diskussionen zu leiten, hilft mir in meiner täglichen Arbeit insbesondere das Training im ,Feedbackgeben' sehr weiter.“

\section{Peter Laier (Karlsruhe)}

„Der Kurs hat mir Wissen vermittelt über die Grundlagen des Lernens und umfangreiches Wissen um das Lehren in unterschiedlichen Modalitäten gegeben. Das ging von praktischen Dingen wie Zeitmanagement bis hin zu komplexen, interaktiven Inhalten wie Moderation von Diskussionsrunden. Ich konnte im Live Event in sehr angenehmer Atmosphäre auch erstmals eine direkte Beurteilung über meine Fähigkeiten erhalten als Vortragender, Leiter einer Diskussionsrunde und Tischinstruktor. Auch konnte ich lernen, selbst strukturiert Feedback zu geben. Das Erlernte im FEP macht in Zukunft meine Tätigkeit in den Kursen für mich nicht nur leichter, sondern sicher auch besser.“

\section{Prof. Dr. Andrea Meyer-Lindenberg (München)}

„Der Kurs beginnt bereits 5 Wochen im Voraus mit einer Onlinevorbereitung zu dieser Veranstaltung, die 2-3 Stunden in der Woche bedarf. Das ist zwar manchmal etwas schwierig im klinischen Alltag gewesen, aber man lernt schon bei dieser Vorbereitung einiges und Teile davon werden für das Face-to-Face Event benötigt. Der Kurs selbst war sehr gut strukturiert und organisiert. Ich konnte aus den verschiedenen Abschnitten des Kurses, die man sowohl allein als auch im Team erarbeiten musste, so einiges Neues lernen, über das man sich vorher keine wirklichen Gedanken gemacht hat. Der Kurs war zudem sehr kurzweilig, weil man zu jeder Zeit in die Aktivitäten eingebunden war. Ich kann diesen Kurs eigentlich jedem, der in AO-Kurse (oder auch andere Fortbildungen) eingebunden ist und sie als Vortragender oder Instruktor mitmachen möchte, sehr empfehlen.“

Bibliografie

DOI https://doi.org/10.1055/s-0043-109137

OP-JOURNAL 2017; 33: 84-86 @ Georg Thieme Verlag KG Stuttgart · New York ISSN 0178-1715 